\title{
Resultados en el corto y mediano plazo de la reparación endovascular de aneurismas de la aorta abdominal y arterias ilíacas*
}

\author{
Drs. JUAN BOMBIN F. ${ }^{1,2}$, ALEJANDRO KOTLIK A. ${ }^{1,2}$, JAIME FERNÁNDEZ V. ${ }^{1,2}$, \\ Tecn. Méd. IVER ZEGARRA I. ${ }^{1}$
}

Servicio de Cirugía, Hospital Dr. Eduardo Pereira.

2 Departamento de Cirugía, Universidad de Valparaíso.

Valparaíso, Chile.

Abstract

\section{Endovascular repair of abdominal aortic aneuriysms. Experience in 28 patients}

Background: Endovascular repair of aortic aneurysms is less invasive than the traditional surgical approach. Aim: To evaluate the early and mid-term results of aorto-iliac aneurysm endovascular repair. Material and Methods: Analysis of 28 patients aged 59 to 86 years (22 males), subjected to an endovascular repair of aorto-iliac aneurysms between 2007 and 2012. Results: The follow up period of patients ranged from 1 to 65 months. A tri-modular bifurcated prosthesis was installed in 25 patients. A bi-modular bifurcated prosthesis was installed in one patient, an aorto uni iliac prosthesis was installed in one patient; in other patient, a straight endoprosthesis was installed after an abdominal visceral disconnection. In cases of associated iliac aneurysms, unilateral iliac embolization was carried out in seven patients and bilateral embolization in one patient. In one case, the sealing of the hypogastric artery was achieved leaning the prosthetic branch on the hypogastric artery ostium. One patient died 31 days after the operation due to multiple organ failure. One patient died 24 months after surgery due to a gallbladder cancer and other patient died 36 months after surgery due to a rectal cancer. The long-term follow-up showed the presence of type 2 endo-leaks in two patients. The aneurysm decreased in size in $83 \%$ of patients after 2 years of follow-up. In five patients, a non-progressive laminar parietal thrombosis inside the endoprosthesis, was observed in the immediate postoperative period. It was initially treated with oral anticoagulants. Conclusions: Endovascular repair of aorto-iliac aneurysms is safe and effective in patients with appropriate anatomical conditions, if a skilled surgical team is available.

Key words: Aortic aneurysm, endovascular repair, complications.

*Recibido el 22 de junio de 2012 y aceptado para publicación el 30 de abril de 2013.

No existe ningún interés ni soporte financiero o comercial en este manuscrito.

El autor (Dr. Juan Bombin F.) durante la ejecución del estudio perteneció al Servicio de Cirugía del Hospital Dr. Eduardo Pereira de Valparaíso y al Departamento de Cirugía de la Universidad de Valparaíso.

Correspondencia: Dr. Juan Bombin F.

14 Norte 571, oficina 411. Viña del Mar, Chile.

drbombin@gmail.com 


\section{Resumen}

Objetivo: Evaluar los resultados iniciales y en el mediano plazo de la reparación endovascular de los aneurismas del sector aorto-ilíaco. Material y Método: Revisión de una serie clínica de pacientes operados en forma consecutiva por vía endovascular en el Hospital Dr. Eduardo Pereira de Valparaíso por aneurismas del sector aorto-ilíaco desde 2007 al 2012 con seguimiento actualizado. Resultados: Se trata de 28 pacientes (22 varones), una edad promedio de 72,5 años (rango 59-86) y un seguimiento promedio de 27,4 meses (rango 1-65 meses). Los pacientes fueron seleccionados de acuerdo a la anatomía aorto-ilíaca y su estado general. A 25 pacientes se les instaló una prótesis bifurcada tri-modular, a un paciente una prótesis bifurcada con sólo dos módulos, a otro paciente una prótesis aorto uni ilíaca, y a una paciente una endoprótesis recta luego de una desconexión visceral abdominal. Un paciente fallece a los 31 días después de operado por falla orgánica múltiple y otros dos pacientes fallecen a los 24 meses a causa de un cáncer vesicular y a los 36 meses por un cáncer rectal. El seguimiento alejado evidenció ausencia de endofugas tipo 1, 3 ó 4 y una endofuga tipo 2 en dos pacientes, que no han requerido ninguna intervención. Conclusión: La reparación endovascular de los aneurismas del sector aorto-ilíaco es segura en el corto y mediano plazo, en pacientes con las condiciones anatómicas apropiadas en un centro quirúrgico adecuadamente equipado.

Palabras clave: Aneurisma, aorta abdominal, arterias ilíacas, reparación endovascular.

\section{Introducción}

En 1991 Parodi introduce la reparación endovascular en el tratamiento de los aneurismas de la aorta, técnica quirúrgica radicalmente diferente y menos invasiva que el tratamiento clásico abierto ${ }^{1}$. La reparación endovascular de un aneurisma aórtico consiste en la colocación de una endoprótesis impermeable dentro de la luz de la aorta aneurismática a través de pequeñas incisiones en las arterias femorales en la zona inguinal, haciendo que el flujo sanguíneo pase dentro de la endoprótesis hacia distal excluyendo y despresurizando el aneurisma, evitando así su crecimiento y la ruptura. La endoprótesis se autosustenta a la pared aórtica con la fuerza radial de un esqueleto de stent metálico, que logra evitar fugas dentro del saco aneurismático ${ }^{2,3}$ y se instala con mucha precisión bajo visión radioscópica simple y con sucesivas inyecciones intra-arteriales de un medio de contraste.

Inicialmente las endoprótesis eran artesanales y permitían sólo el tratamiento de pequeños aneurismas aórticos puros, pero han mejorado progresivamente y ahora son dispositivos fabricados por la industria que logran resolver casos cada vez más complejos de aneurismas del sector aorto-ilíaco ${ }^{4}$.

En esta comunicación damos a conocer nuestra experiencia en la introducción de este costoso y complejo método quirúrgico en un hospital público y los resultados en el corto y mediano plazo de una serie clínica en el Hospital Dr. Eduardo Pereira dependiente del Servicio de Salud Valparaíso-San Antonio.

\section{Material y Método}

Serie clínica de 28 casos consecutivos de pacientes portadores de aneurismas del sector aorto-ilíaco tratados mediante una reparación endovascular desde el año 2007 al año 2012, con seguimiento actualizado, en el Hospital Dr. Eduardo Pereira (HEP) de Valparaíso.

\section{Selección de pacientes}

Consideramos para reparación endovascular a los pacientes portadores de un aneurisma de la aorta abdominal con un diámetro de $5 \mathrm{~cm}$ o mayor ${ }^{5}$, además se incluye 3 pacientes con diámetros de $4,7 \mathrm{~cm}$, mujeres o aneurismas con crecimiento reciente. En el caso de la arteria ilíaca, la consideramos aneurismática con un diámetro de $3 \mathrm{~cm}$ o mayor. Los pacientes firman un consentimiento informado y aceptan tener un control médico periódico y permanente. El riesgo quirúrgico general debe ser no mayor que el requerido para cualquier cirugía aórtica.

Además, se deben cumplir condiciones anatómicas en el aneurisma aórtico para que sea compatible con la instalación de una endoprótesis (Tabla 1).

\section{Casuística}

Consiste en 28 pacientes ( 22 hombres) con un promedio de edad de 72,3 años (rango 59-86 años). La mayoría de los pacientes fueron asintomáticos, pero tres presentaron síntomas, uno con dolor abdominal y lumbar intenso por ruptura aneurismática contenida, otro paciente consultó por embolización distal a repetición en una extremidad y una paciente que consultó por dolor pélvico vago persistente tenía aneurismas ilíacos comunes e hipogástricos bilaterales, sin aneurisma en la aorta. El aneurisma era aórtico infrarrenal puro en 18 casos, uno era aórtico visceral supra-renal, siete aneurismas eran aortoilíacos y 2 casos presentaban aneurismas ilíacos con ausencia de aneurisma aórtico. El diámetro promedio del aneurisma aórtico al momento pre-operatorio era de 6,33 cm (rango 4,7-9,8 cm). 
Tabla 1. Condiciones anatómicas de un aneurisma aórtico infrarrenal para reparación endovascular

- Acceso femoral adecuado

- Diámetro del lumen de los vasos ilíacos mayor de $7 \mathrm{~mm}$

- Ausencia de tortuosidad y angulación severa en arterias ilíacas

- Escasas o ausencia de calcificaciones arteriales

- Largo de las arterias ilíacas comunes mayor de $20 \mathrm{~mm}$

- Cuello aórtico mayor de $15 \mathrm{~mm}$ de largo

- Ausencia de trombosis mural en el cuello

- Angulación del cuello menor a $60^{\circ}$ respecto al cuerpo aórtico

- Cuello recto, no cónico

- Diámetro del lumen distal del aneurisma aórtico mayor de $20 \mathrm{~mm}$

Tabla 2. Intervenciones o procedimientos quirúrgicos previos

\begin{tabular}{|ll|}
\hline $\begin{array}{l}\text { Colocación de malla de polipropileno sobre } \\
\text { el aneurisma hace años y luego dos intentos } \\
\text { "abiertos" fallidos }\end{array}$ & 1 \\
\hline $\begin{array}{l}\text { Cirugía clásica de aneurisma aórtico infrarrenal y años } \\
\text { después desconexión de otro aneurisma visceral }\end{array}$ & 1 \\
\hline Nefrectomía derecha por cáncer & 1 \\
Endoprótesis en aneurisma torácico & 1 \\
Embolización hipogástrica previa & 7 \\
\hline Fístula intestinal, hernia incisional, abdomen hostil & 1 \\
\hline Puente fémoro-poplíteo por aneurisma poplíteo & 1 \\
\hline
\end{tabular}

Todos los pacientes eran portadores de alguna patología asociada, destacando la hipertensión arterial en 15 pacientes, tabaquismo en 12 , cardiopatía coronaria en 6 y diabetes mellitus en 6 . Varios pacientes tenían intervenciones quirúrgicas previas importantes relacionadas (Tabla 2).

\section{Reparación endovascular del aneurisma}

Se efectuó siempre bajo anestesia general, colocando una endoprótesis bifurcada trimodular en 25 pacientes, una prótesis bifurcada con sólo dos módulos en un paciente portador de un aneurisma aórtico sacular, una endoprótesis aorto uni ilíaca más puente fémoro-femoral supra-púbico en un paciente con una incurvación ilíaca muy severa, y una endoprótesis recta cónica en una paciente portadora de un aneurisma visceral con una desconexión visceral previa $^{6-9}$. En los aneurismas aorto-ilíacos e ilíacos puros se efectuó embolización hipogástrica un mes antes en 7 casos y simultánea con la colocación de la endoprótesis aórtica en 2 casos, completando una embolización hipogástrica bilateral en una paciente. En otro paciente el sellado de la arteria hipogástrica se logró apoyando la rama protésica en el ostium de la arteria hipogástrica.

\section{Seguimiento}

Todos los pacientes operados fueron sometidos a controles post-operatorios clínicos y con angio scanner al mes, 6 meses y luego controles anuales. Los resultados de los exámenes radiológicos fueron evaluados por el equipo vascular y un radiólogo especializado, y las imágenes y los datos fueron guardados en archivos informáticos.

\section{Resultados}

\section{Resultados inmediatos}

En todos los pacientes se logró la colocación completa de la endoprótesis. En el intra-operatorio se verificó la ausencia de endofugas tipo I; en un paciente se verificó una endofuga tipo 2 desde una arteria mesentérica inferior permeable. No fueron necesarios procedimientos secundarios correctores. En el paciente en que se usó una endoprótesis aorto uni ilíaca se complementó con un puente fémorofemoral, habitual en estos casos. Un paciente falleció a los 31 días a causa de una insuficiencia renal, cardíaca y falla respiratoria; era portador de una cardiopatía coronaria y sufrió la obstrucción de una rama protésica con insuficiencia arterial aguda de la extremidad requiriendo un puente fémoro-femoral de urgencia.

\section{Resultados alejados}

El seguimiento alejado promedio de la serie fue de 27,4 meses (rango 1-65 meses). De doce pacientes que completaron dos años de seguimiento, se observó una disminución del tamaño del aneurisma aórtico en 10, pero se mantuvo el tamaño en dos pacientes, en ausencia de endofugas. El diámetro transversal máximo aórtico promedio inicial de $6,04 \mathrm{~cm}$ disminuyó a 4,69 $\mathrm{cm}$ al término de los dos años de seguimiento alejado (Figura 1). En los angio scanner de control se observó endofuga tipo 2 en dos pacientes desde la arteria mesentérica inferior y otro desde una arteria lumbar, sin encontrar crecimiento aneurismático en ellos. No hubo migración, alteración o infección de la endoprótesis. En los pacientes tratados mediante embolización hipogástrica no hubo disfunción eréctil, ni signos de claudicación glútea. Observamos en 5 pacientes $(17,8 \%)$ una trombosis mural laminar dentro del cuerpo princi- 


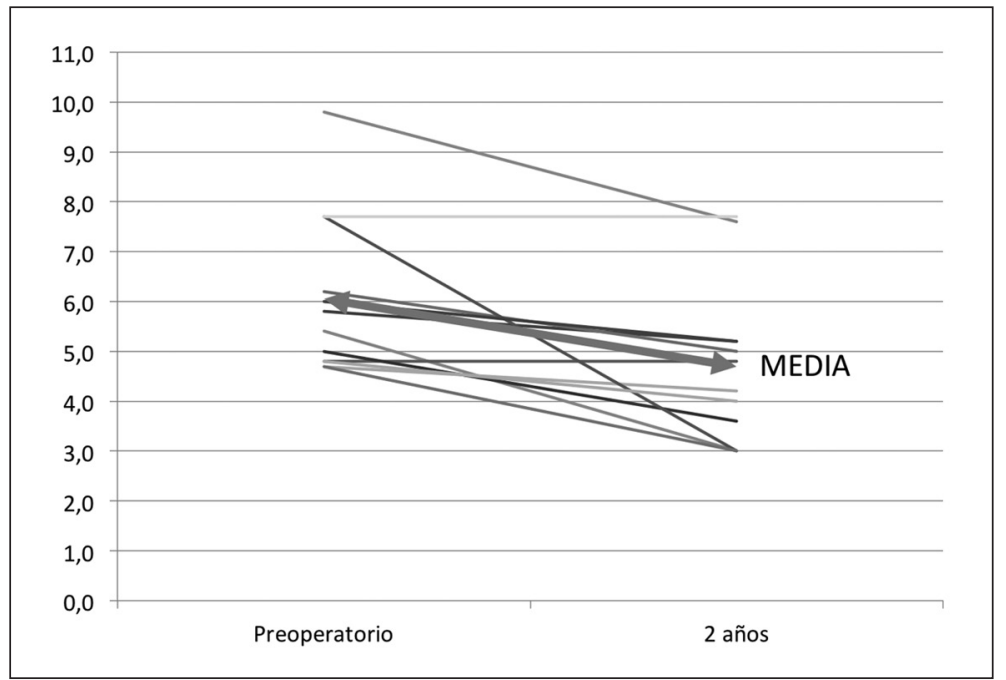

Figura 1. Diámetro transversal preoperatorio del aneurisma y luego de 2 años de seguimiento. pal de la endoprótesis asintomática que se observó desde el primer control radiológico y que se ha mantenido sin alteraciones en los controles radiológicos posteriores (Figura 2). Dos de esos pacientes fueron tratados por este motivo durante un año con anticoagulantes orales sin variación de las imágenes. Los 5 pacientes siguen asintomáticos hasta hoy. En el seguimiento alejado un paciente falleció a los 24 meses a causa de un cáncer vesicular y otro paciente falleció a los 36 meses por un cáncer rectal.

\section{Discusión}

Luego del advenimiento de la cirugía endovascular aórtica hace unos 20 años ${ }^{1}$, han seguido ocurriendo cambios importantes en el tratamiento de los aneurismas aórticos abdominales y torácicos ${ }^{3}$, úlceras penetrantes, disecciones aórticas complicadas y patología vascular traumática, revolución terapéutica similar a la ocurrida años antes con la introducción de la cirugía laparoscópica. Actualmente hay un creciente número de reparaciones endovasculares de aneurismas de la aorta abdominal o torácica en todo el mundo y en nuestro país, que han desplazado en muchos casos la reparación clásica abierta ${ }^{3,10,11}$. Ha ocurrido también una progresiva reorientación de los cirujanos especialistas hacia las técnicas endovasculares.

Entre las competencias necesarias para este tipo de reparación de un aneurisma creemos que es muy importante saber interpretar los exámenes imagenológicos actuales, los diversos softwares y las reconstrucciones vasculares tridimensionales, con el objeto de poder medir exactamente la anatomía

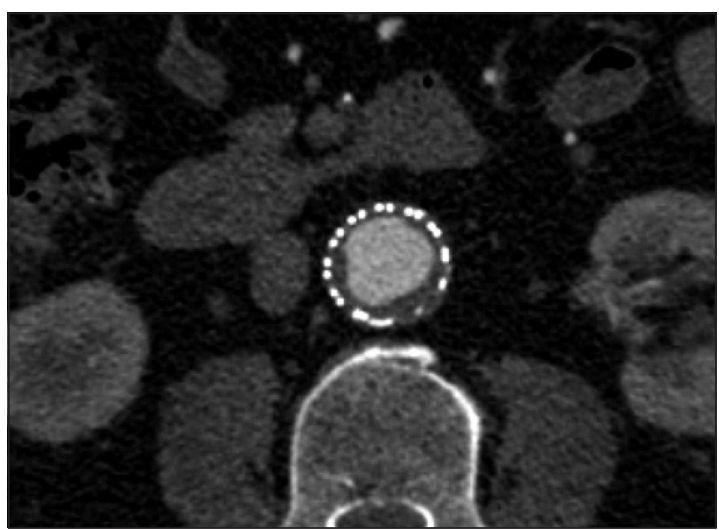

Figura 2. Trombosis mural laminar dentro del cuerpo principal de la endoprótesis aórtica.

aórtica y seleccionar de ese modo los pacientes y las endoprótesis a utilizar, planificar adecuadamente la intervención endovascular y realizar después el seguimiento alejado. Esta selección permitirá descartar aquellos pacientes con anatomía desfavorable a esta técnica, evitando complicaciones.

Es fundamental la formación continua de los equipos médicos por la incesante mejoría tecnológica de la industria. El papel de la industria en el entrenamiento y asesoría puede ser discutida, sin embargo, los hospitales públicas no disponen de suficiente presupuesto para capacitación en este campo de innovación constante y creemos que sería muy difícil incluso poder iniciar la cirugía endovascular aórtica prescindiendo completamente de alguna asociación con la industria proveedora.

Nuestra casuística inicial corresponde a la de- 
nominada "curva de aprendizaje" y refleja la experiencia ya comunicada en la literatura en cuanto a las características de la población, procedimientos realizados y sus resultados en el corto y mediano plazo en experiencias iniciales y en grandes series clínicas $^{3,10-12}$. La ausencia de complicaciones relacionadas a la prótesis en el mediano plazo reafirma las cualidades de las endoprótesis actuales en el tratamiento endovascular de los aneurismas aórticos abdominales en los casos adecuadamente seleccionados, de acuerdo a las condiciones anatómicas exigidas por el fabricante ${ }^{13}$. Sin embargo, hemos observado en cinco pacientes la presencia de una trombosis laminar mural dentro del cuerpo principal de la endoprótesis que no impide el flujo sanguíneo hacia distal, que aparece ya en los primeros controles de Angio scanner y se mantiene en el tiempo. A dos pacientes le hemos indicado tratamiento anticoagulante oral durante un tiempo prolongado sin lograr ningún cambio en la trombosis mural intra-protésica, al igual que otros tres pacientes no tratados con anticoagulantes. Desconocemos la causa de la aparición de esta trombosis mural laminar intra-protésica, sin embargo, hasta este momento su evolución ha sido benigna.

\section{Referencias}

1. Parodi JC, Palmaz J, Barone HD. Transfemoral intraluminal graft implantation for abdominal aortic aneurysms. Ann Vasc Surg. 1991;5:491-9.

2. Maeso-Lebrun J, Clará A, Escudero-Rodríguez JR, Gesto-Castromil R, Gómez-Palonés FJ, Riambau-Alonso V, et al. Tratamiento endovascular de la patología aneurismática de la aorta abdominal. Angiología 2007;59(supl 1):S3-S28.

3. Mertens R, Valdés F, Krämer A. Tratamiento endovascular del aneurisma de aorta. Rev Med Chile 2004;56:311 .

4. Torella F. Effect of improvement endograft implantation for abdominal aortic aneurysms. N England J Med. 2004:351:1607-18.

5. The UK Small Aneurysm Trial Participants. Mortality results for randomised controlled trial of early surgery of ultrasonographic surveillance for small abdominal aortic aneurysms. Lancet 1998;352:1649-55.

6. Metha M, Veith FJ, Ohki T, Cynamon J, Goldstein K, Suggs WD, et al. Unilateral and bilateral hypogastric artery interruption during aortoiliac aneurysm repair in 154 patients: a relatively innocuous procedure. J Vasc Surg. 2001;33:S27-S32.

7. Bratby MJ, Munneke GM, Loosemore TM, Loftus I, Thompson MM, Morgan RA. How safe is bilateral internal iliac artery embolization prior to EVAR? Cardiovasc Intervent Radiol. 2008;31:246-53.

8. Wyers MC, Schermerhorn ML, Fillinger MF, Powell RJ, Rzucidlo EM, Walsh DB, et al. Internal iliac occlusion without coil embolization during endovascular abdominal aortic aneurysm repair. J Vasc Surg. 2002;36:113845 .

9. Bombin J, Espíndola M, Kotlik A, Zegarra I. Tratamiento de aneurisma de la aorta abdominal con revascularización visceral extra-anatómica y posterior colocación de endoprótesis. Técnicas Endovasculares 2010;13:3482-7.

10. De Bruin JL, Baas AF, Buth J, Prinssen M, Verhoeven EL, Cuypers PW, et al. Long-term outcome of open or endovascular repair of abdominal aortic aneurysm (DREAM Study) N Engl J Med. 2010;362:1881-9.

11. EVAR Trial Participants. Endovascular aneurysm repair versus open repair in patients with abdominal aortic aneurysm (EVAR trial 1): randomised controlled trial. Lancet 2005;365:2179-86.

12. Valdés F, Seitz J, Fava M, Kramer A, Mertens R, Espíndola $\mathrm{M}$, y cols. Tratamiento del aneurisma aórtico abdominal por vía endovascular: Experiencia inicial. Rev Med Chile 1998;126:1206-15.

13. Abraham CZ, Chuter TA, Reilly LM, Okuhn SP, Pethan LK, Kerlan RB, et al. Abdominal aortic aneurysm repair with the Zenith stent graft: Short to midterm results. J Vasc Surg. 2002;36:217-25. 